\title{
Dexamethasone and betamethasone protect against lipopolysaccharide-induced brain damage in neonatal rats
}

\author{
Yi Pang', Lir-Wan Fan', Baoying Zheng', Leigh R. Campbell', Zhengwei Cai' and Philip G. Rhodes'
}

INTRODUCTION: The aim of this study was to test whether dexamethasone (Dex) and betamethasone (Beta), two of the most commonly used corticosteroids, protect against lipopolysaccharide (LPS)-induced white matter damage and neurobehavioral dysfunction.

METHODS: LPS or sterile saline was injected into the brain white matter of rat pups at postnatal day 5 (P5), and Dex or Beta was given intraperitoneally to the rat pups $1 \mathrm{~h}$ before the LPS microinjection. Brain inflammatory response, brain damage, and myelination were examined at P6, P8, and P14. Neurobehavioral tests were performed from P3 through P22.

RESULTS: Our results demonstrate that Dex and Beta markedly diminish the LPS-induced brain inflammatory response, restore myelin basic protein (MBP) expression, and alleviate lateral ventricle dilation. Both corticosteroids demonstrate significant protection against most LPS-induced behavioral deficits, including those in rearing, vibrissa-elicited forelimb-placing, beam walking, learning, and elevated plus-maze test. Of note, only Beta improved the locomotion and stereotype dysfunction. In contrast to their beneficial effects, neither drug prevented LPSinduced delay in body weight gain from P6 through P21.

DISCUSSION: Our study suggests that if their adverse effects are minimized, corticosteroids may be the potential candidate drugs to prevent brain damage in premature infants.

$\mathbf{P}$ eriventricular leukomalacia (PVL) is the most common form of brain injury in preterm infants and results in high mortality and morbidity in this group of infants. The etiology of PVL is still unclear, but there is considerable evidence that maternal infection/inflammation and hypoxia-ischemia are two major contributory factors (1-3). We have previously reported PVL-like neuropathological changes (4) and neurobehavioral deficits in the neonatal rat (5) after intracerebral delivery of lipopolysaccharide (LPS). Later, we further showed that suppression of microglial activation by minocycline is protective against LPS-induced oligodendrocyte (OL) damage (6). As brain inflammation is also associated with neuronal damage in hypoxia-ischemia animal models (7), anti-inflammation is one of the plausible strategies to prevent and/or treat PVL in the future.

Corticosteroids are potent anti-inflammatory drugs and have been used clinically to treat septic shock and other inflammatory diseases for half a century. Antenatal corticosteroid therapy has also been used to reduce the incidence of respiratory distress syndrome and other complications of preterm birth (8). In a recent large-population clinical study, Lee et al. (9) reported that antenatal exposure of both dexamethasone (Dex) and betamethasone (Beta) have tended to reduce the incidence of PVL and intraventricular hemorrhage. Dex and Beta are two corticosteroids used to accelerate lung maturation in the perinatal period. Therefore, the aim of this study was to test whether these two corticosteroids may protect against brain white matter damage and neurobehavioral deficits following LPS exposure.

\section{RESULTS}

Dex and Beta Suppressed the LPS-Induced Inflammatory Response in the Rat Brain

Consistent with our previous observations, LPS induced an acute inflammatory response characterized by microglia/macrophage activation and polymorphonuclear leukocyte (PMN) infiltration after 24-h treatment (Figure 1). The increased ED1 ${ }^{+}$cells and infiltrated PMNs were primarily detected in the periventricular white matter, and to a lesser extent, the subventricular zone, the cerebral cortex, and the striatum. Inducible nitric oxide synthasepositive (iNOS ${ }^{+}$) cells were found exclusively in the periventricular white matter area in the LPS-treated rat brain (Figure $\mathbf{2 b}$, shown in low-power magnification). Double-labeling immunohistochemistry showed that iNOS is expressed by both $\mathrm{CD}^{+} 3^{+}$ PMNs and $\mathrm{ED}^{+}$microglia, but not by glial fibrillary acidic protein-positive astrocytes or neuron/glial antigen $2\left(\mathrm{NG}^{+}\right)$ OL progenitor cells (Figure $2 \mathbf{e}-\mathbf{h}$ ). The inflammatory response was significantly suppressed by both Dex and Beta treatments, as shown by a decreased number of PMNs (Figure 1g,h) and ED1 ${ }^{+}$ microglia/macrophages (Figure $1 \mathbf{k}, \mathbf{l}$ ). iNOS ${ }^{+}$cells were significantly reduced by both corticosteroid treatments (control: $0 \pm 0$; LPS: $27.7 \pm 4.1$; Dex: $1.0 \pm 0.6$; Beta: $1.0 \pm 0.5$; mean \pm SEM). It appears that Dex and Beta have very similar efficacy in suppressing brain inflammation. No differences between Dex or Beta treatment without LPS and the control were noted.

Dex and Beta Prevented LPS-Induced Loss of Pre-OLs and Myelination Deficits

$\mathrm{OL}$ marker $\mathrm{O}^{+}$pre-OLs are the major OL lineage in the P5 rat brain (10); therefore, we used $\mathrm{O} 4$ immunohistochemistry 

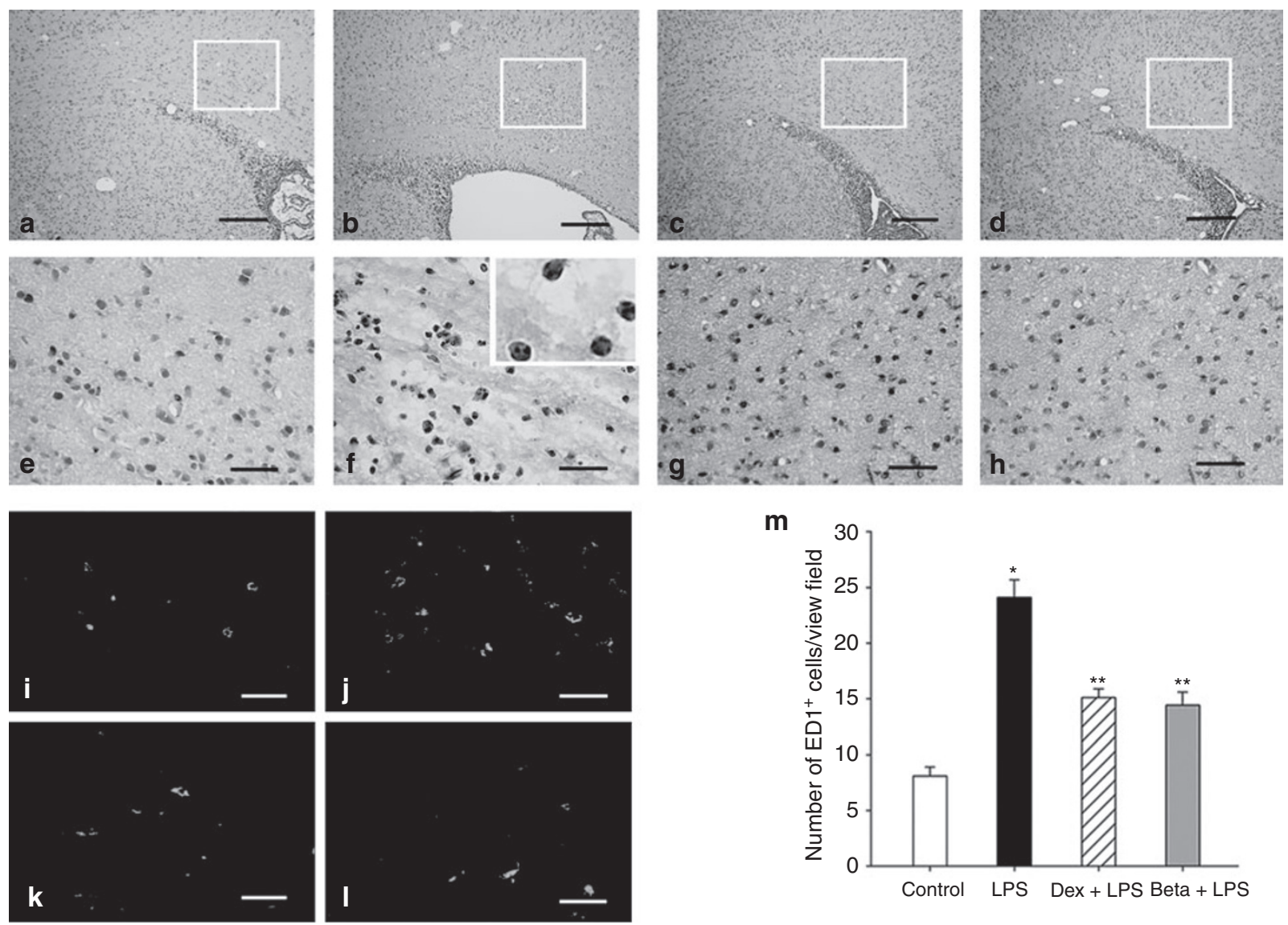

m

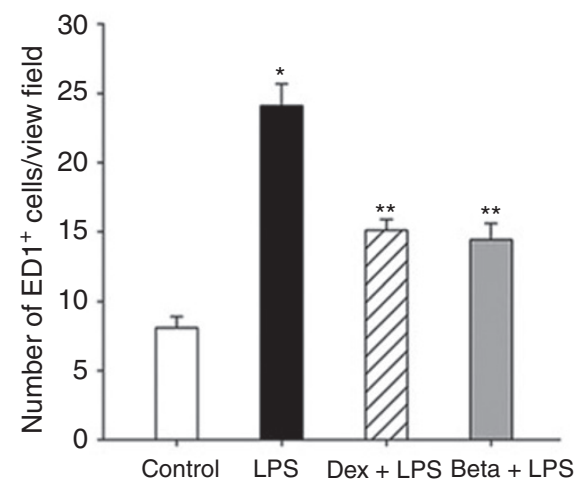

Figure 1. Dex and Beta suppress LPS-induced PMN infiltration and microglia activation in the P6 rat brain. (a-h) Hematoxylin and eosin staining for examination of PMN infiltration. No PMNs were found in the control rat brain (a and e), whereas LPS induced a marked recruitment of PMN into brain parenchyma, especially in the white matter (b and $\mathbf{f})$. The typical PMNs show horseshoe-like nuclei that can be readily identified (magnified in the insert in (f)). Both Dex (c and $\mathbf{g}$ ) and Beta ( $\mathbf{d}$ and $\mathbf{h}$ ) significantly reduced PMN infiltration. Images in (e-h) are higher magnifications of white boxes in the corresponding images from $(\mathbf{a}-\mathbf{d})$. (i-I) ED1 immunostaining for examination of microglia activation. ED $1^{+}$cells (green) were primarily found in the periventricular white matter in both the control (i) and LPS-treated rat brain (j), but their numbers were significantly increased after LPS treatment (quantitative counting data shown in ( $\mathbf{m})$ ). Both Dex (k) and Beta (I) significantly suppressed microglia activation. Sections were counterstained with propidium iodide (red) (color image available online). Data shown are mean \pm SEM from eight animals. Bar $(\mathbf{a}-\mathbf{d})=250 \mu \mathrm{m}$. Bar $(\mathbf{e}-\mathbf{I})=100 \mu \mathrm{m}$. ${ }^{*} P<0.01$ vs. control; ${ }^{* *} P<0.01$ vs. LPS. Beta, betamethasone; Dex, dexamethasone; LPS, lipopolysaccharide; PMN, polymorphonuclear leukocyte.

to quantify changes of pre-OLs. Three days after LPS treatment, the number of $\mathrm{O}^{+}$pre-OLs was significantly diminished. Some $\mathrm{O} 4^{+}$cells appeared to be undergoing degeneration, as shown by the condensed $\mathrm{O} 4$ immunostaining in the soma and by fragmented processes (Figure $3 \mathbf{b}$ ). Later at P14, myelin basic protein (MBP) immunostaining showed a marked hypomyelination in LPS-treated rats (Figure 3g,h), as compared with the well-developed myelination in the control group (Figure $3 \mathrm{e}, \mathrm{f}$ ). The number of pre-OLs (Figure $3 \mathrm{~m}$ ) and the amount of MBP immunostaining (Figure 3n) were significantly increased in both Dex and Beta treatment groups. No differences between Dex or Beta without LPS and the control groups were noted.

\section{Dex and Beta Attenuated LPS-Induced Lateral Ventricle Dilation}

One of the most consistent pathological features in LPS-treated rat brain is the bilateral dilation of lateral ventricles $(4,5)$. As shown in Table 1 , the lateral ventricles were significantly dilated in LPS-treated rat brain as compared with the control. Both Dex and Beta significantly decreased the ventricle dilation by LPS.
Dex appears to be slightly more effective than Beta, although this difference is not statistically significant $(P>0.05)$.

\section{Dex and Beta Improved LPS-Induced Behavioral Deficits}

The long-term neurological functions of rats were evaluated by a battery of behavioral tests. Locomotor activity increased with age until P15 in all groups. LPS-treated rats showed hyperactivity on P15 as compared with the controls (Figure 4a). Beta but not Dex significantly prevented LPS-induced hyperactivity. Stereotypy (Figure 4b) and rearing activity (Figure 4c) were significantly increased in LPS-exposed rats (only observed at P21 and P14, respectively) relative to the controls. Both corticosteroid treatments showed recovery on rearing activity, but only Beta showed improvement on stereotypy activity.

Motor coordination was evaluated by a beam-walking test. LPS-treated rats showed a significant increase in performance latency as compared with the control rats (Figure 4d, tested on both $3.5 \times 3.5$ and $1.4 \times 1.4 \mathrm{~cm}^{2}$ beams). Sensorimotor function, as evaluated by the vibrissa-elicited forelimb-placing test on P20, was also impaired in LPS-treated rats (Figure 4e, 

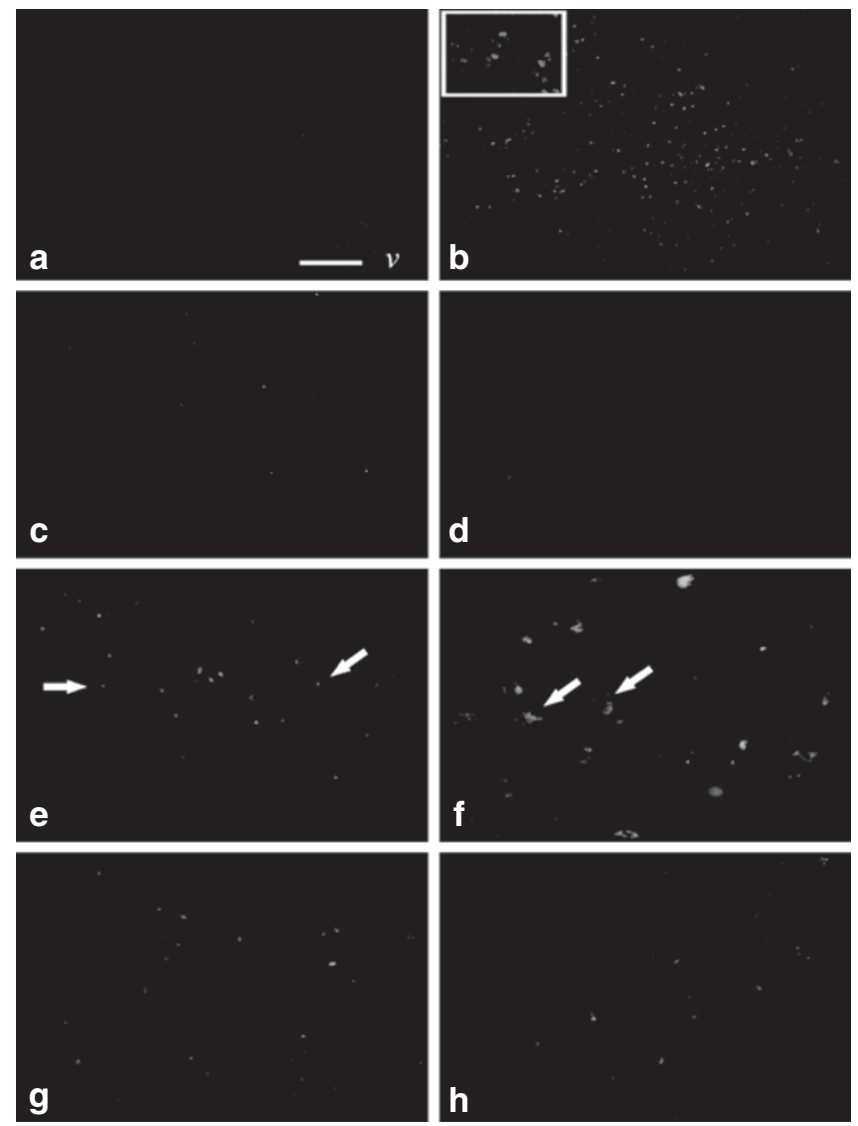

Figure 2. Dex and Beta suppress LPS-induced iNOS expression in the rat brain. Extensive iNOS ${ }^{+}$cells were found in the LPS-treated ((b), the white box highlights iNOS ${ }^{+}$cells in higher magnification) but not the control rat brain (a), $24 \mathrm{~h}$ after treatment. The induction of iNOS was significantly reduced by Dex (c) and Beta (d) treatment. Double-labeling shows that the majority of iNOS $^{+}$ cells (green) co-localize with CD43 ${ }^{+}$PMNs (red in (e)) and ED1 ${ }^{+}$microglia/ macrophages (red in (f)), but not GFAP ${ }^{+}$astrocytes (red in (g)) or neuron/glial antigen 2 (NG2+) OL progenitor cells (red in (h)) (color image available online). Arrows in (e) and (f) delineate co-labeled cells. Bar $(\mathbf{a}-\mathbf{d})=250 \mu \mathrm{m}$. Bar $(\mathbf{e}-\mathbf{h})=$ $100 \mu \mathrm{m}$. Beta, betamethasone; Dex, dexamethasone; GFAP, glial fibrillary acidic protein; iNOS, inducible nitric oxide synthase; LPS, lipopolysaccharide; $\mathrm{OL}$, oligodendrocyte; PMN, polymorphonuclear leukocyte; v, lateral ventricle.

significant decrease in success rate). Both corticosteroid treatments showed significant improvement on these two tests.

The plus-maze test showed that LPS-treated animals exhibit lower anxiety-like behavior, as demonstrated by a significantly increased number of entries into the open field (Figure 4f). The passive avoidance test showed that the number of electric foot shocks required for retaining the rats on the safe board significantly increased in LPS-treated pups at P20, suggesting that learning ability was impaired in these animals. LPS also tended to reduce the retention latency to step down from the board the next day (P21) as compared with the control group, but did not reach a statistically significant level (Figure $4 \mathrm{~g}$ ). Both Dex and Beta treatments significantly prevented LPSinduced lowered anxiety-like behavior and learning deficits.

\section{Dex and Beta Failed to Improve LPS-Induced Body Weight Loss}

LPS treatment resulted in an immediate arrest in body weight gain in the first $24 \mathrm{~h}$. Body weight started to increase at $48 \mathrm{~h}$ and continued to increase with a similar trajectory as that of the control rats, but there never was a catch-up growth as compared with the control. Neither Dex nor Beta treatment prevented the LPS-induced delay in body weight gain. Dex or Beta alone $(n=8)$ delayed normal weight gain, in a similar pattern to that of rats treated with LPS (Figure $4 \mathrm{~h}$ ).

\section{DISCUSSION}

This study showed that both Dex and Beta treatment prevented LPS-induced brain damage in the neonatal rat. This is presumed to be associated with their powerful anti-inflammatory properties.

Our previous in vivo studies have suggested that activated microglia play a central role in LPS-induced brain damage in neonatal rats $(5,6)$. In line with these findings, our current study demonstrates that the neuroprotection against LPS-induced brain damage by Dex and Beta is associated with a marked decrease in the inflammatory response. LPS upregulates iNOS expression in both activated microglia and PMNs, and this is significantly suppressed by steroid treatment (Figure 2). Therefore, the neuroprotective effects of Dex and Beta treatment may be attributed, at least partially, to their suppression of NO and oxidative species. The role of NO in LPS-induced OL damage has been recently demonstrated by an in vitro study that showed that $\mathrm{NO}$ and subsequent reactive nitrogen species produced by LPS-activated microglia can lead to acute pre-OL lysis, while a delayed OL degeneration is mediated by proinflammatory cytokines such as tumor necrosis factor $a$ (11). The role of PMNs in inflammatory brain damage is less studied as compared with microglia, but they likely contribute to a substantial extent by producing toxic factors such as proinflammatory cytokines (12) and reactive oxygen species (13). Therefore, we speculate that suppression of inflammatory mediators and reactive nitrogen species may underlie the major neuroprotective mechanism by the corticosteroids used in this study.

Lateral ventricle dilation is frequently observed in infants with PVL. Of note, we have consistently documented enlarged lateral ventricles in our LPS intracerebral injection model, and we have used this neuropathology as a general indicator of brain damage $(4,5)$. It is generally thought that an increase in intraventricular pressure, or loss of surrounding tissue and a subsequent space-filling by ventricular enlargement are two potential causes of ventricle dilation.

Although white matter is predominantly damaged in our LPS model, we recently found that gray matter, e.g., the dopaminergic system, is also compromised (14). The many behavioral deficits in LPS-exposed rats are likely due to both white and gray matter damage. Our goal for neurobehavioral tests was to utilize these tools as general measures for assessing neuroprotection by corticosteroids. Most of the behavioral deficits were ameliorated by both steroids, except that the locomotor and stereotypy activity were selectively improved by treatment with Beta and not Dex. The reason for this disparity between these two corticosteroids, which showed very similar antiinflammatory effects, is not known at this time. However, we 

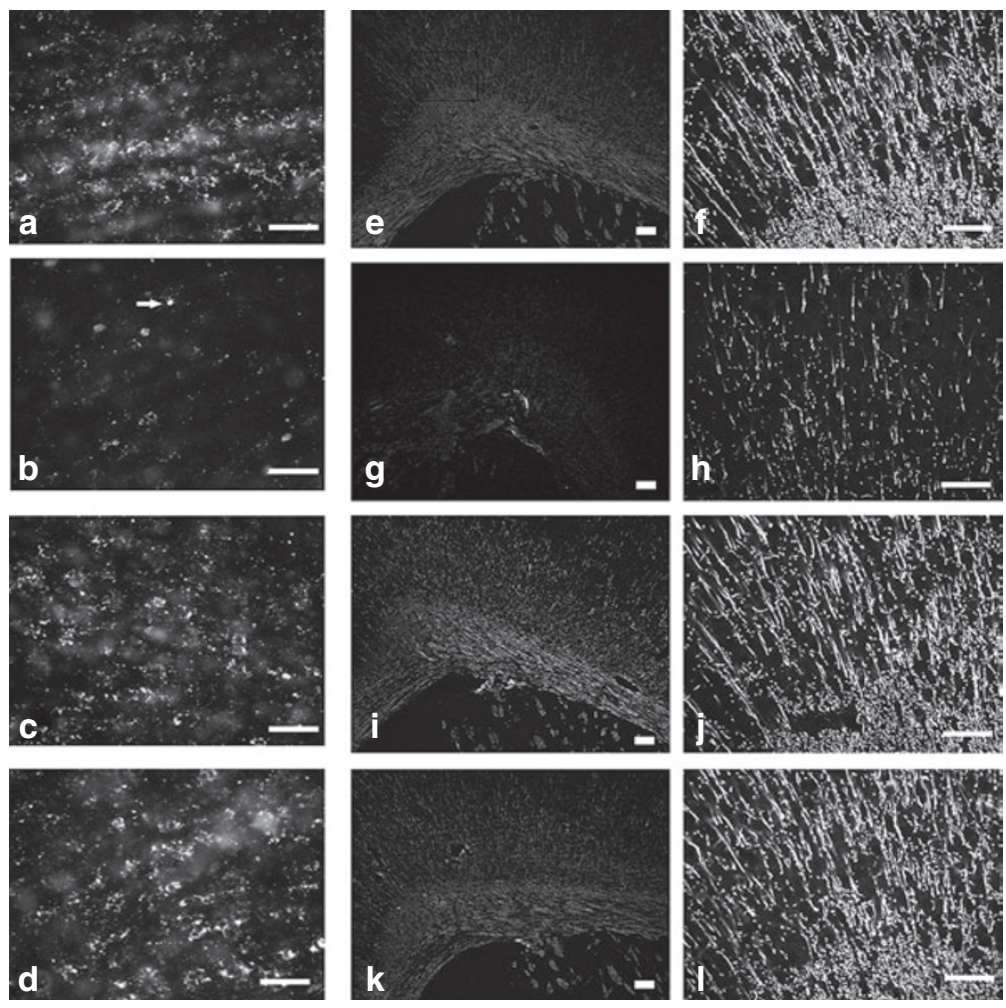
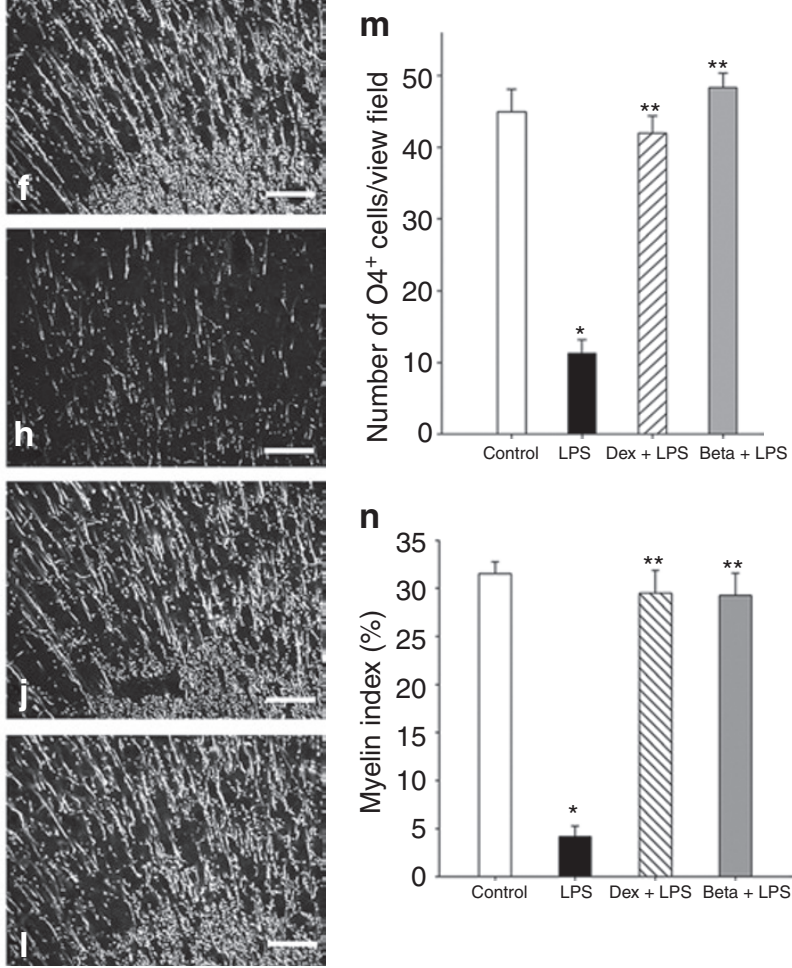

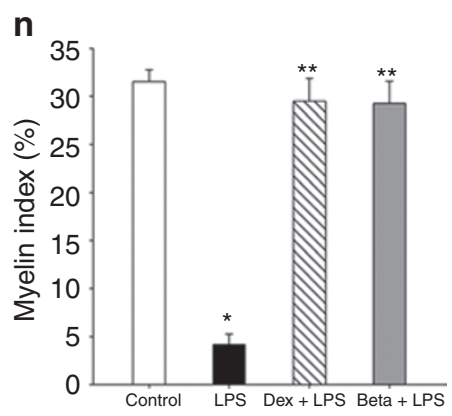

Figure 3. Dex and Beta attenuate LPS-induced loss of pre-OLs at P8 and hypomyelination at P14. (a-d) OL marker O4 immunohistochemistry. Numerous pre-OLs were revealed by $\mathrm{O} 4$ immunostaining in the white matter of the control rat brain (a), whereas their numbers were significantly reduced by LPS exposure (b). Some OLs appeared to undergo degeneration (arrow). Dex (c) and Beta (d) significantly prevented loss of O4 ${ }^{+}$cells in the white matter after LPS treatment. (e-I) MBP immunohistochemistry. At P14, LPS induced severe hypomyelination as shown by reduced areas and intensity of MBP immunoreactivity $(\mathbf{g}, \mathbf{h})$, which is in contrast to the rather well-myelinated brain white matter track in the controls (e,f). Dex (i,j) and Beta ( $\mathbf{k}, \mathbf{I})$ significantly attenuated LPS-induced hypomyelination. Bar $=100 \mu \mathrm{m}$. (m) Quantification of $\mathrm{O}^{+}$cell counting. (n) Myelin index. Data are shown as mean \pm SEM from eight animals. ${ }^{*} P<0.01$ vs. control; ${ }^{* *} P<0.01$ vs. LPS. Beta, betamethasone; Dex, dexamethasone; LPS, lipopolysaccharide; MBP, myelin basic protein; OL, oligodendrocyte; P, postnatal day.

Table 1. Lateral ventricle size index in the neonatal rat brain

\begin{tabular}{|c|c|c|}
\hline \multirow[b]{2}{*}{ Treatment } & \multicolumn{2}{|c|}{ Ventricle size index (\%) } \\
\hline & Left ventricle & Right ventricle \\
\hline Control & $0.49 \pm 0.21$ & $0.42 \pm 0.18$ \\
\hline LPS & $4.85 \pm 1.03^{*}$ & $3.85 \pm 0.61^{*}$ \\
\hline Dex + LPS & $1.09 \pm 0.57^{* *}$ & $0.75 \pm 0.42^{* *}$ \\
\hline Beta + LPS & $1.19 \pm 0.21^{* *}$ & $0.99 \pm 0.63^{* *}$ \\
\hline \multicolumn{3}{|c|}{$\begin{array}{l}\text { Changes in the lateral ventricle size in the P15 rat brain are estimated by a ventricle size } \\
\text { index, which is defined as the ratio between the area of each ventricle and the area of } \\
\text { the whole-brain section measured by Quantity One software. The left side was injected } \\
\text { with LPS or sterile saline. Data are shown as mean } \pm \text { SD and analyzed by one-way } \\
\text { ANOVA followed by post hoc Tukey test. Seven rats were used in each group. }\end{array}$} \\
\hline \multicolumn{3}{|c|}{ Beta, betamethasone; Dex, dexamethasone; LPS, lipopolysaccharide; P, postnatal day. } \\
\hline${ }^{*} P<0.01 \mathrm{vs} . \mathrm{Cc}$ & the same side of ve & \\
\hline
\end{tabular}

can speculate that this may be associated with the adverse neurological effects of Dex (see the following), rather than lack of neuroprotection.

Although Dex- and Beta-mediated neuroprotection may be largely due to their anti-inflammatory activity, it may also result from their direct protection of OLs. For instance, in vitro studies have demonstrated that corticosteroids are protective against cytokine-induced blockage of survival and differentiation of OL progenitor cells (15) and cell death (16). If this is also true in vivo, it can explain why Dex and Beta treatments show nearly complete protection against brain damage in our current study. Of note, Ikeda et al. (17) have reported that upregulation of endogenous corticosteroids by LPS is associated with neuroprotection against subsequent hypoxicischemic brain damage (known as LPS preconditioning), which further supports this notion.

Dex and Beta are the only two corticosteroids currently used antenatally to accelerate fetal lung maturation, especially in those pregnant women with imminent delivery of an infant at 24 to $34 \mathrm{wk}$ of gestation. During the past decade, concerns have been raised regarding their potential adverse effects on development (particularly about Dex). There are numerous studies suggesting that Beta has less adverse effects than Dex when used prenatally. For instance, in a large population study conducted by the National Institute of Child Health and Human Development Neonatal Research Network, it was reported (9) that Beta was associated with a reduced risk for neonatal death and with trends of decreased risk for other adverse neonatal outcomes as compared with Dex. Later, a study from the same group further showed that antenatal Beta exposure was associated with reduced risks, whereas Dex exposure was associated with increased risks of hearing impairment and neurodevelopmental 

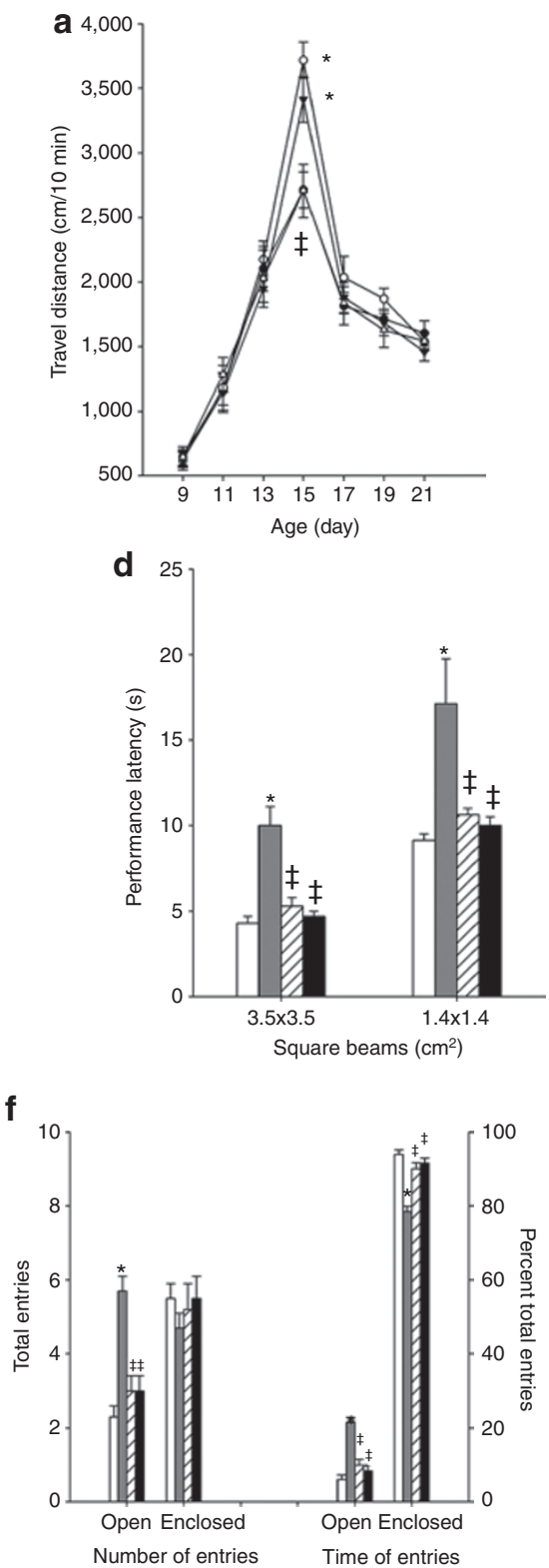
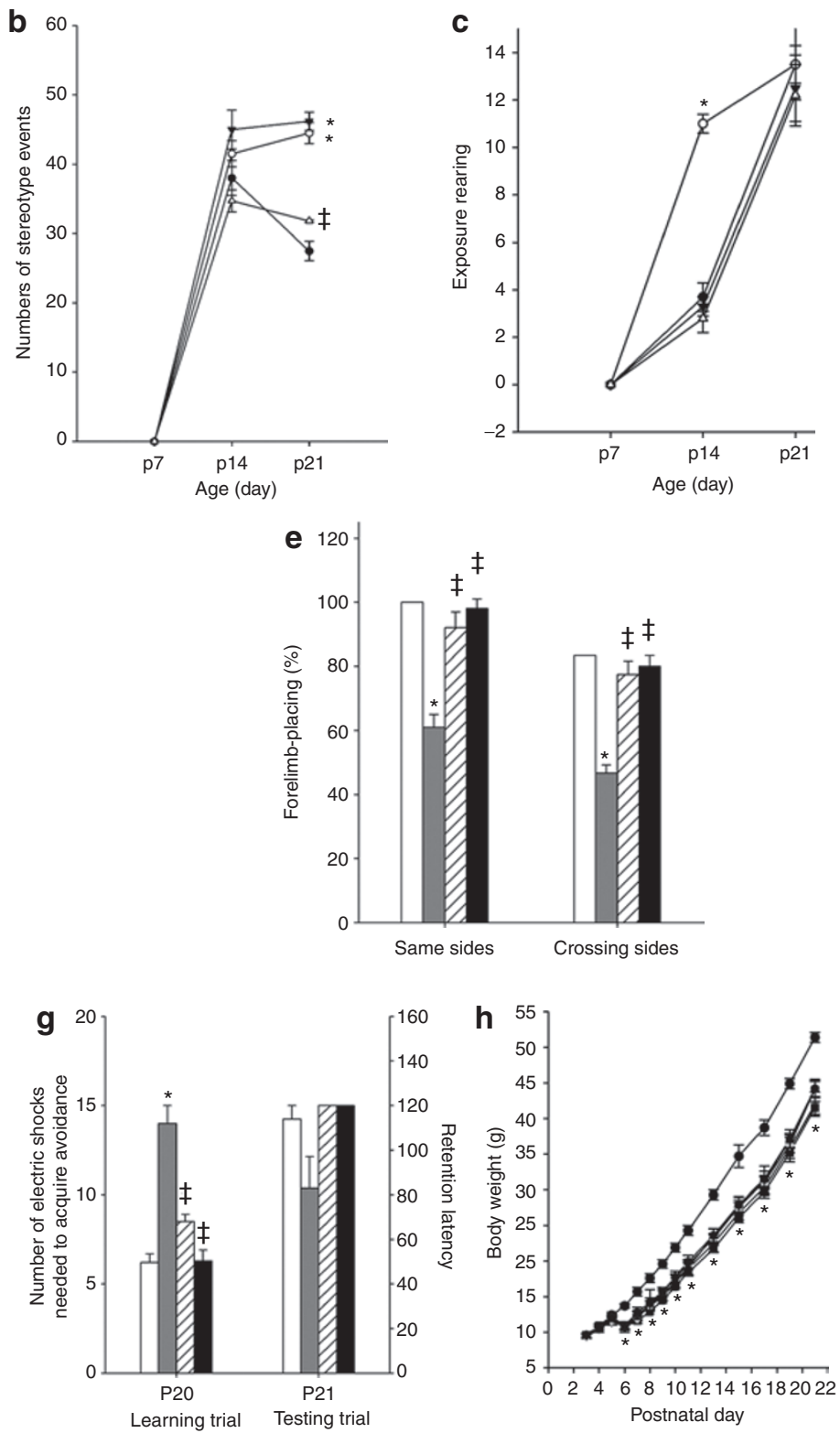

Figure 4. Effects of corticosteroid treatment on LPS-induced neurobehavioral deficits and body weight loss. (a-c) Locomotor activity tests. LPS significantly increased (a) locomotor activity at P15, (b) stereotype behavior at P21, and (c) exposure rearing at P14. Both corticosteroids reversed LPS-induced increase in exposure rearing, but only Beta significantly attenuated LPS-induced increase in locomotor and stereotype activity. (d,e) Motor function tests. LPS-treated rats showed impaired motor function, as indicated by increased time during beam walking (d). LPS also impaired the sensory motor function as shown by the reduced success rate of vibrissa-elicited forelimb-placing (e). Both steroids showed recovery in beam walking and vibrissa-elicited forelimb-placing tests. (f) Anxiety test. LPS induced a lower anxiety-like behavior (significantly increased number of entries and times spent in the open field during the elevated maze test) in the rats, which was significantly ameliorated by Dex and Beta treatment. (g) Learning and memory test. LPStreated rats showed deficits in learning during the passive avoidance test. The learning deficits were significantly ameliorated by Dex and Beta. It should be noted that the time spent on the safe platform by Dex- and Beta-treated rats all exceeded $120 \mathrm{~s}$ (the cutoff value); their retention latencies were simply assigned as $120 \mathrm{~s}$, and therefore no standard errors were obtained from these two groups. (h) Body weight changes. Neither of the corticosteroids protect against LPS-induced arrest in body weight gain. Further studies showed that Dex and Beta themselves cause delayed body weight gain similar to that by LPS. Black circle, control; open circle, LPS; inverted triangle, Dex + LPS; triangle, Beta + LPS; open bar, control; gray bar, LPS; striped bar, Dex + LPS; black bar, Beta + LPS. Beta, betamethasone; Dex, dexamethasone; LPS, lipopolysaccharide; P, postnatal day.

impairments, and a decreased likelihood of unimpaired status, as compared with the control group (18). Consistent with these clinical data, our animal study shows that while providing similar efficacy in suppressing inflammation, Beta is more effective than Dex in decreasing LPS-induced behavioral deficits.
One of the adverse effects of corticosteroids on development is a reduced body weight and/or head circumference (19). In line with these clinical reports, our animal study showed that both corticosteroids significantly reduced body weight in the presence or absence of LPS. It should be noted that the severity of these adverse effects correlates with the dose of corticosteroids used 
(20). Although a single dose usually is given prenatally, multiple doses sometimes are used prenatally without much confirmed benefit (8). In this study, the doses of Dex and Beta used are clinically relevant but at the high end. It is possible that by reducing the currently used doses of Dex and Beta, adverse effects can be minimized. As the most common reason for preterm labor is maternal infection (which can also result in fetal cerebral inflammation), neonates with evidence of active infection may need to be excluded from corticosteroid therapy. In brief, we demonstrate the neuroprotection of corticosteroids against inflammatory brain damage, suggesting that they may be potential candidates in preventing brain damage in premature infants.

\section{METHODS}

\section{Chemicals}

LPS (055:B5), Dex, and Beta were obtained from Sigma Chemical (St. Louis, MO). Antibodies used were obtained from the following sources: O4, ED1, glial fibrillary acidic protein, and MBP from Millipore (Temecula, CA); CD43 from AbD Serotec (Raleigh, NC), and iNOS from Cell Signaling Technology (Danvers, MA).

\section{Animal Surgery and Treatment}

Myelination in rats occurs exclusively after birth (21), and the developmental stage of OLs in the rat brain at $\mathrm{P} 2-\mathrm{P} 7$ is roughly equivalent to that in the human at 24-32 gestational wk (10), a developmental window with a high incidence of PVL. Therefore, we used a P5 rat model in which PVL-like brain injury has been consistently documented $(4,5)$. Briefly, Sprague-Dawley rat pups at P5 were anesthetized with isoflurane ( $4 \%$ induction, $1.5 \%$ maintenance) and placed in a stereotaxic apparatus with an adapter for neonatal rats (David Kopf, Tujunga, CA). The intracerebral injection was performed using a $10-\mu$ precise syringe (World Precision Instruments, Sarasota, FL) in the following coordination: $1.0 \mathrm{~mm}$ posterior and $1.0 \mathrm{~mm}$ lateral to the bregma, and $2.5 \mathrm{~mm}$ deep to the skull surface. LPS $(1 \mu \mathrm{g} / \mathrm{animal})$ or the vehicle (for the control) at the volume of $2 \mu \mathrm{l}$ was injected into the left brain hemisphere over a period of $5 \mathrm{~min}$. Dex or Beta (both used at $0.5 \mathrm{mg} / \mathrm{kg}$ body weight) was given to rat pups intraperitoneally $1 \mathrm{~h}$ before LPS injection. The doses are equivalent to clinical uses to treat premature infants.

To minimize variations in body and brain size, the litter size was adjusted to 10 pups per litter. The experimental procedure was approved by the Institutional Animal Care and Use Committee at the University of Mississippi Medical Center and, in addition, was in accordance with the guidelines of the National Institutes of Health on the care and use of laboratory animals.

\section{Histologic Examination and Immunohistochemistry}

At P6, P8, and P14, rat brains were fixed by transcardiac perfusion with $4 \%$ paraformaldehyde. Frozen sections $(10 \mu \mathrm{m})$ or free-floating sections $(40 \mu \mathrm{m}$, for $\mathrm{O} 4$ immunohistochemistry) were consecutively prepared at the level of the bregma. Hematoxylin and eosin-stained sections were examined under a light microscope for any alterations in histopathology. Immunohistochemistry was performed using a standard protocol as previously described $(4,5)$. The final concentrations of the antibodies were diluted as follows: O4 (1:100), ED1 (1:200), MBP (1:100), CD43 (1:200), and iNOS (1:100). Images were captured with a CCD camera (Oly-750; Olympus, Pittsburgh, PA) and superimposed using Adobe Photoshop (version 7.0) software, if necessary.

To compare the size of lateral ventricles, hematoxylin and eosinstained sections at the bregma level were scanned by a densitometer (Bio-Rad, Richmond, CA) to acquire digital images for area measurement, which was done using Quantity One software (Bio-Rad, version 4.5.2). The areas $\left(\mathrm{cm}^{2}\right)$ occupied by the left and right ventricles, as well as that by the whole-brain section were outlined and measured. Ventricle size index was calculated as a ratio of the area of each ventricle to that of the whole-brain hemispheres.

\section{Quantification of Immunohistochemistry Data}

Three consecutive brain sections in each animal were used for cell counting. $\mathrm{O}^{+}, \mathrm{EDl}^{+}$, and $\mathrm{iNOS}^{+}$cells were counted in the entire periventricular white matter area (including corpus callosum and cingular white matter). The mean value was calculated from three slides of the same animal and averaged. MBP immunohistochemistry was quantified using ImageJ software version 1.45p (National Institutes of Health). Images of MBP immunostaining (primarily at the corpus callosum) from three consecutive sections at the bregma level were acquired under a low-power microscopic view $(\times 10)$. The ratio of the area occupied by MBP immunostained fibers to that of the entire image frame was calculated and was defined as the myelin index (see Figure 3). Cell counting and myelin index assessment were performed in a blinded manner in that the individuals who performed the procedure were unaware of the test conditions.

\section{Behavioral Tests}

The developmental test battery used was based on the behavioral tests for neurotoxicity $(22,23)$ and were performed from P3 to P21. Behavioral tests were performed by an individual who was unaware of the test conditions. Body weights of rat pups were recorded daily (from P3 to P21). Eight animals were included in each treatment group.

Locomotor activity. Locomotor activity was measured by the Video Tracking System-SMART (2000 San Diego Instruments, San Diego, CA). Pups were placed in the activity chambers in a quiet room with a dimmed light. The total distance traveled by the animals was recorded during a 10 -min testing period.

The numbers of rearing events including exposure rearing response (body inclined vertically with hindpaws on the floor of the activity cage and forepaws on the wall of the cage) and sniffing-air response (rearing in the open area of the active cage) were counted. The summation of exposure rearing and sniffing-air responses reflects vertical activity, which has been used apart from locomotion and has been reported as a reliable criterion for assignment of rats into groups during their exposure to novelty (24).

Stereotypy. The stereotyped behaviors including standing (on all four feet, essentially motionless, and no active sniffing), grooming (washing the face or any other parts of its body with the forepaws, with the mouth generally in contact with the body), scratching (raising of hindpaws to touch any part of its body), head-swinging (standing on all four feet and moving its head from side to side), sniffing (sniffing parts of the walls or floor of the apparatus), and freezing (standing on all four feet in a freezing position, completely inactive, i.e., head oriented forward and eyes fixed at a point of the upper side of the cage) were quantified during the first $5 \mathrm{~min}$ of testing period.

Elevated plus-maze test. The plus-maze consists of two open arms (30 $\times 5 \times 0.25 \mathrm{~cm})$ and two enclosed arms $(30 \times 5 \times 10 \mathrm{~cm})$ emanating from a common central platform $(5 \times 5 \mathrm{~cm})$ to form a plus shape. The anxiety-related behaviors were recorded by a video camera for a period of $5 \mathrm{~min}$ on P19. The parameters used for data analysis were the numbers of open or enclosed arm entries (arm entry was defined as placement of all four paws into an arm) and the time the animals spent in the various sections of the maze (open arms, center, and enclosed arms).

Beam-walking test. Motor coordination and balance were assessed by measuring the ability of the animals to traverse a narrow beam to reach an enclosed safety platform. The time spent on the beam for each pup to traverse the beam and join its littermates was recorded, and the cutoff time was set at $60 \mathrm{~s}$. The test was performed on P20.

Vibrissa-elicited forelimb-placing test. This test uses stimulation of the rat's vibrissae to trigger a placing response to measure forelimb-placing deficit (25). Rats use their vibrissae to gain bilateral information about the proximal environment, and this information is integrated between the hemispheres. The test was performed on P21. The animal was gently held by its torso, allowing the forelimbs to hang free. 
Independent testing of each forelimb was induced by gently brushing vibrissae of the corresponding side on the edge of a tabletop once per trial for 10 trials. The percentage of trials in which the rat successfully placed its forepaw onto the tabletop was recorded for each side. Intact animals placed the forelimbs of both sides quickly onto the countertop, with $100 \%$ success in all variants of this test. If an animal struggled during testing, the data were not included in the overall analysis.

Passive avoidance (learning and memory tests). The passive avoidance procedure consists of two sessions designed to test learning and memory. In the first session (P20), rats were trained to learn how to avoid an electric shock. The number of shocks required to retain an individual animal on a safe board for 2 min was recorded as a measure of acquisition of passive avoidance. The second session was carried out $24 \mathrm{~h}$ after the first session (P21). The rat was placed on the safe board, and steel rods were not connected to the electric shock generator. The retention latency, i.e., the time elapsed before the rat stepped down to the grid floor, was recorded as a measure of the retention of passive avoidance. If the rat did not step down to the grid floor within $2 \mathrm{~min}$, a ceiling score of $2 \mathrm{~min}$ was assigned.

\section{Statistics}

One-way ANOVA followed by post hoc Tukey test was used to determine statistical significance among treatments, and the level of significance was set at $P<0.05$.

\section{STATEMENT OF FINANCIAL SUPPORT}

This work was supported by National Institutes of Health-funded grant NS54278 and by funds from the Department of Pediatrics, University of Mississippi Medical Center.

\section{REFERENCES}

1. Leviton A, Dammann O. Coagulation, inflammation, and the risk of neonatal white matter damage. Pediatr Res 2004;55:541-5.

2. Dammann O, Leviton A. Inflammation, brain damage and visual dysfunction in preterm infants. Semin Fetal Neonatal Med 2006;11:363-8.

3. Khwaja O, Volpe JJ. Pathogenesis of cerebral white matter injury of prematurity. Arch Dis Child Fetal Neonatal Ed 2008;93:F153-61.

4. Pang Y, Cai Z, Rhodes PG. Disturbance of oligodendrocyte development, hypomyelination and white matter injury in the neonatal rat brain after intracerebral injection of lipopolysaccharide. Brain Res Dev Brain Res 2003;140:205-14.

5. Fan LW, Pang Y, Lin S, et al. Minocycline reduces lipopolysaccharideinduced neurological dysfunction and brain injury in the neonatal rat. J Neurosci Res 2005;82:71-82.

6. Cai Z, Lin S, Fan LW, Pang Y, Rhodes PG. Minocycline alleviates hypoxicischemic injury to developing oligodendrocytes in the neonatal rat brain. Neuroscience 2006;137:425-35.

7. Vexler ZS, Yenari MA. Does inflammation after stroke affect the developing brain differently than adult brain? Dev Neurosci 2009;31:378-93.

8. Cavalieri RL, Cohen WR. Antenatal steroid therapy: have we undervalued the risks? J Matern Fetal Neonatal Med 2006;19:265-9.

9. Lee BH, Stoll BJ, McDonald SA, Higgins RD; National Institute of Child Health and Human Development Neonatal Research Network. Adverse neonatal outcomes associated with antenatal dexamethasone versus antenatal betamethasone. Pediatrics 2006;117:1503-10.

10. Craig A, Ling Luo N, Beardsley DJ, et al. Quantitative analysis of perinatal rodent oligodendrocyte lineage progression and its correlation with human. Exp Neurol 2003;181:231-40.

11. Pang Y, Campbell L, Zheng B, Fan L, Cai Z, Rhodes P. Lipopolysaccharideactivated microglia induce death of oligodendrocyte progenitor cells and impede their development. Neuroscience 2010;166:464-75.

12. Cassatella MA, Gasperini S, Russo MP. Cytokine expression and release by neutrophils. Ann N Y Acad Sci 1997;832:233-42.

13. Grau AJ, Berger E, Sung KL, Schmid-Schönbein GW. Granulocyte adhesion, deformability, and superoxide formation in acute stroke. Stroke 1992;23:33-9.

14. Fan LW, Tien LT, Zheng B, et al. Dopaminergic neuronal injury in the adult rat brain following neonatal exposure to lipopolysaccharide and the silent neurotoxicity. Brain Behav Immun 2011;25:286-97.

15. Mann SA, Versmold B, Marx R, et al. Corticosteroids reverse cytokineinduced block of survival and differentiation of oligodendrocyte progenitor cells from rats. J Neuroinflammation 2008;5:39.

16. Melcangi RC, Cavarretta I, Magnaghi V, Ciusani E, Salmaggi A. Corticosteroids protect oligodendrocytes from cytokine-induced cell death. Neuroreport 2000;11:3969-72.

17. Ikeda T, Yang L, Ikenoue T, Mallard C, Hagberg H. Endotoxin-induced hypoxic-ischemic tolerance is mediated by up-regulation of corticosterone in neonatal rat. Pediatr Res 2006;59:56-60.

18. Lee BH, Stoll BJ, McDonald SA, Higgins RD; National Institute of Child Health and Human Development Neonatal Research Network. Neurodevelopmental outcomes of extremely low birth weight infants exposed prenatally to dexamethasone versus betamethasone. Pediatrics 2008;121:289-96.

19. Yeh TF, Lin YJ, Lin HC, et al. Outcomes at school age after postnatal dexamethasone therapy for lung disease of prematurity. N Engl J Med 2004;350:1304-13.

20. Abbasi S, Hirsch D, Davis J et al. Effect of single versus multiple courses of antenatal corticosteroids on maternal and neonatal outcome. Am J Obstet Gynecol 2000;182:1243-9.

21. Watson RE, Desesso JM, Hurtt ME, Cappon GD. Postnatal growth and morphological development of the brain: a species comparison. Birth Defects Res B Dev Reprod Toxicol 2006;77:471-84.

22. Altman J, Sudarshan K, Das GD, McCormick N, Barnes D. The influence of nutrition on neural and behavioral development. 3. Development of some motor, particularly locomotor patterns during infancy. Dev Psychobiol 1971;4:97-114.

23. Hermans RH, Hunter DE, McGivern RF, Cain CD, Longo LD. Behavioral sequelae in young rats of acute intermittent antenatal hypoxia. Neurotoxicol Teratol 1992;14:119-29.

24. Antoniou K, Papathanasiou G, Panagis G, Nomikos GG, Hyphantis T, Papadopoulou-Daifoti $\mathrm{Z}$. Individual responses to novelty predict qualitative differences in d-amphetamine-induced open field but not reward-related behaviors in rats. Neuroscience 2004;123:613-23.

25. Woodlee MT, Asseo-García AM, Zhao X, Liu SJ, Jones TA, Schallert T. Testing forelimb placing "across the midline" reveals distinct, lesion-dependent patterns of recovery in rats. Exp Neurol 2005;191:310-7. 